\title{
Constitutive CD40 Signaling in Dendritic Cells Limits Atherosclerosis by Provoking Inflammatory Bowel Disease and Ensuing Cholesterol Malabsorption
}

\author{
Pascal Kusters, ${ }^{*}$ Tom Seijkens, ${ }^{*}$ Christina Bürger, ${ }^{\dagger}$ Bart Legein, ${ }^{\dagger}$ Holger Winkels, ${ }^{\dagger}$ Marion Gijbels, ${ }^{* \ddagger}$ Christian Barthels, \\ Remy Bennett, ${ }^{*}$ Linda Beckers, ${ }^{*}$ Dorothee Atzler, ${ }^{\dagger \uparrow}$ Erik Biessen, ${ }^{\dagger \|}$ Thomas Brocker, ${ }^{\S}$ Christian Weber, ${ }^{\dagger}$ Norbert Gerdes, ${ }^{\dagger * *}$ and \\ Esther Lutgens ${ }^{* \dagger}$ \\ From the Department of Medical Biochemistry, * Experimental Vascular Biology, Academic Medical Center, University of Amsterdam, Amsterdam, the \\ Netherlands; the Institutes for Cardiovascular Prevention (IPEK $)^{\dagger}$ and Immunology, ${ }^{\S}$ Ludwig-Maximilian University of Munich, Munich, Germany; the \\ Department of Pathology, ${ }^{\ddagger}$ Experimental Vascular Pathology, Cardiovascular Research Institute Maastricht, University of Maastricht, Maastricht, \\ the Netherlands; the Walther-Straub-Institut for Pharmacology and Toxicology, ${ }^{\circledR}$ Ludwig Maximilians University, Munich, Germany; the Institute \\ for Molecular Cardiovascular Research (IMCAR)," Klinikum RWTH Aachen, Aachen, Germany; and the Division of Cardiology, Pulmonology, \\ and Vascular Medicine,** Medical Faculty, University Hospital Düsseldorf, Düsseldorf, Germany
}

Accepted for publication August 22, 2017.

Address correspondence to Esther Lutgens, M.D., Ph.D., Department of Medical Biochemistry, Room K1-110, Meibergdreef 15, $1105 \mathrm{AZ}$ Amsterdam, the Netherlands. E-mail: e.lutgens@amc.uva.nl.

\begin{abstract}
The costimulatory molecule CD40 is a major driver of atherosclerosis. It is expressed on a wide variety of cell types, including mature dendritic cells (DCs), and is required for optimal T-cell activation and expansion. It remains undetermined whether and how CD40 on DCs impacts the pathogenesis of atherosclerosis. Here, the effects of constitutively active CD40 in DCs on atherosclerosis were examined using low-density lipoprotein-deficient $\left(\mathrm{Ldll}^{-/}\right)$bone marrow chimeras that express a transgene containing an engineered latent membrane protein 1 (LMP)/CD40 fusion protein conferring constitutive CD40 signaling under control of the DC-specific CD11c promoter (DC-LMP1/CD40). As expected, DC-LMP1/CD40/ $\mathrm{Ldll}^{-/-}$chimeras (DC-LMP1/CD40) showed increased antigen-presenting capacity of DCs and increased Tcell numbers. However, the mice developed extensive neutrophilia compared to CD40wt/Ldll ${ }^{-1-}$ (CD40wt) chimeras. Despite overt T-cell expansion and neutrophilia, a reduction in conventional DC frequency and a dramatic (approximately 80\%) reduction in atherosclerosis was observed. Further analyses revealed that cholesterol and triglyceride levels had decreased by $37 \%$ and $60 \%$, respectively, in DC-LMP1/CD40 chimeras. Moreover, DC-LMP1/CD40 chimeras developed inflammatory bowel disease characterized by massive transmural influx of leukocytes and lymphocytes, resulting in villous degeneration and lipid malabsorption. Constitutive activation of CD40 in DCs results in inflammation of the gastrointestinal tract, thereby impairing lipid uptake, which consequently results in attenuated atherosclerosis. (Am J Pathol 2017, 187: 2912-2919; https://doi.org/10.1016/j.ajpath.2017.08.016)
\end{abstract}

Atherosclerosis is a lipid-driven inflammatory disease characterized by accumulations of lipids and immune cells in the arterial intima. ${ }^{1-4}$ In the past few years, dendritic cells (DCs) were recognized to play an important role in atherogenesis. ${ }^{5}$ DCs are present in atherosclerosis-prone areas of healthy aortas and accumulate at these sites during the onset of atherosclerosis. ${ }^{6}$ Hence, depletion of DCs conferred atheroprotection by reduced foam cell formation and subendothelial lipid deposition. ${ }^{7}$ Upon antigen exposure, DCs up-regulate major histocompatibility complex class II (MHC II) and costimulatory molecules such as CD40, CD80, and CD86. ${ }^{8}$ Antigens are internalized in DCs and subsequently presented via $\mathrm{MHC}$ complexes, thereby activating $\mathrm{T}$ cells. ${ }^{9,10} \mathrm{CD}^{+} \mathrm{T}$ cells,

\footnotetext{
Supported by the Netherlands Organization for Scientific Research VICI grant (E.L.), the Netherlands CardioVascular Research Initiative grant CVON2011-19 (E.L.), Deutsche Forschungsgemeinschaft grants CRC1054B04 (E.L.) and CRC1123-A5 (E.L., N.G., and C.W.), the European Research Council (E.L.), and the EU Horizon 2020 REPROGRAM grant (E.L.).

Disclosures: None declared.
} 

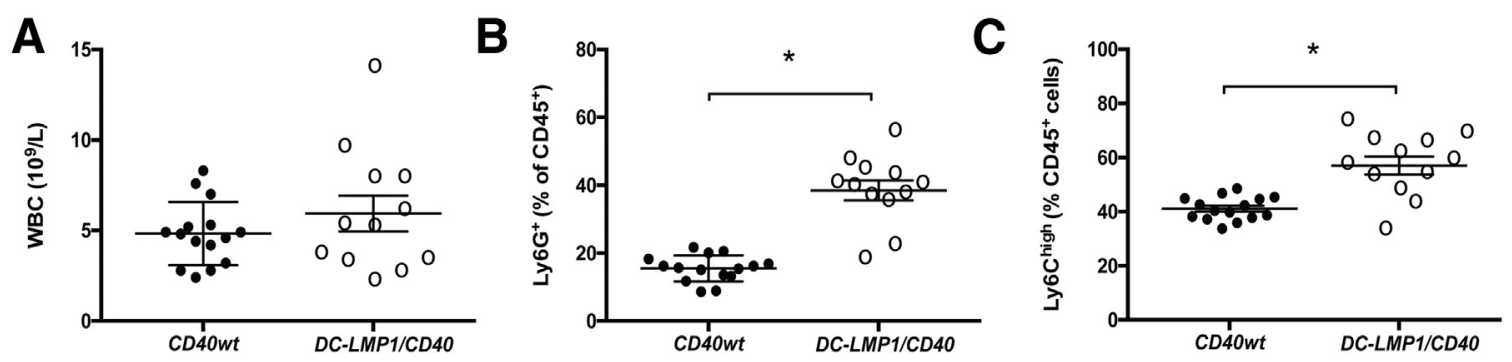

Figure 1 Constitutive CD40 signaling in DCs causes neutrophilia and Ly6 $\mathrm{C}^{\text {high }}$ monocytosis. Hematological and flow cytometric analysis in CD4Owt/Ldlr ${ }^{-/-}$ (CD4Owt) and DC-LMP1/CD40/Ldlr ${ }^{-1-}(D C-L M P 1 / C D 40)$ chimeras of blood leukocyte numbers (A), blood neutrophil counts (Ly6G) (B), and Ly6C high monocyte counts (C). Data represent means \pm SEM. $n=12 D C-L M P 1 / C D 40 / L d l r^{-/}$chimera $(\mathbf{A}-\mathbf{C}) ; n=15$ CD40wt/Ldlr ${ }^{-/}$chimera $(\mathbf{A}-\mathbf{C})$. *P $<0.05$. WBC, white blood count.

particularly Th1 cells, and $\mathrm{CD} 8^{+} \mathrm{T}$ cells are well-known drivers of atherosclerosis. ${ }^{1,3,11}$

Costimulatory molecules are crucial for antigen presentation and priming of $\mathrm{T}$ cells. ${ }^{12,13}$ The costimulatory molecule CD40 is constitutively expressed on DCs and is strongly up-regulated upon encounter of antigen and subsequent DC maturation. Following interaction with T-cell CD40 ligand (CD40L), both $\mathrm{CD}^{+}$and $\mathrm{CD}^{+} \mathrm{T}$ cells expand, polarize, and become effector cells. ${ }^{14}$

Previously, we and others have reported the importance of CD40 and $\mathrm{CD} 40 \mathrm{~L}$ in atherosclerosis. Inhibition of CD40-CD40L interactions not only led to reduced atherosclerosis, but also induced a favorable fibrotic plaque phenotype that only contained few immune cells and small necrotic cores. $^{15-19}$ However, the relative contribution of CD40 expression by DCs to atherosclerosis remains unclear. We hypothesize that DC-CD40 drives atherosclerosis by inducing effector T-cell responses. We thus investigated the role of constitutively active CD40 signaling in DCs in atherosclerosis, using the $D C-L M P 1 / C D 40$ mouse. This mouse expresses a transgene containing a latent membrane protein 1 (LMP)CD40 fusion protein, which confers constitutive activation of CD40 signaling, ${ }^{20,21}$ under the DC-specific CD11c promoter.

\section{Materials and Methods}

\section{Animals}

DC-LMP1/CD40 mice were generated at the LudwigMaximilian University of Munich by breeding Cd11c-cre mice $^{22}$ on a C57BL/6 background to $L M P / C d 4 O^{A S T O P}$ mice, which express a loxP-flanked stop-codon protected latent membrane protein 1 (LMP1)/CD40 chimeric protein utilizing the Rosa26 locus. ${ }^{20,21}$ The LMP1/CD40 fusion protein consists of the signaling domain of CD40 and the transmembrane domain of LMP1. It was previously shown that expression of this chimeric LMP/CD40 fusion protein in B cells leads to constitutive CD40 signaling. ${ }^{20,23}$ Littermates, not expressing the CDIlc-cre transgene, were used as controls (CD40wt mice).

Male C57BL/6, $\mathrm{Ldll}^{-/-}$mice (The Jackson Laboratories, Bar Harbor, ME) $(n=30)$ were bred and housed at the animal facility of the Academic Medical Center,
Amsterdam. The committee for animal welfare of the University of Amsterdam approved all study protocols.

\section{Bone Marrow Transplantation}

DC-LMP1/CD40 and CD40wt mice $(n=4)$ were sacrificed, and bone marrow from femurs and tibias was harvested in ice-cold phosphate-buffered saline, and subsequently resuspended in RPMI 1640 medium containing $5 \mathrm{U} / \mathrm{mL}$ heparin (Heparin Leo). Recipient $\mathrm{Ldll}^{-/-}$mice $(n=30)$ were housed in filter-top cages and received drinking water containing antibiotics (polymyxinB sulfate, $60,000 \mathrm{U} / \mathrm{L}$, and neomycin, $100 \mathrm{mg} / \mathrm{L}$ ) starting 1 week before the bone marrow transplantation. Recipient mice were irradiated on two consecutive days $(2 \times 6 \mathrm{~Gy})$. After the second irradiation, each $\mathrm{Ldll}^{-/-}$recipient received $2 \times 10^{6}$ bone marrow cells of either $D C-L M P 1 / C D 40$ or CD40wt mice i.v. Following a 6-week recovery period, chimeric $\mathrm{Ldll}^{-1-}$ mice were fed a high-cholesterol diet $(0.15 \%$ cholesterol, $16 \%$ fat; Hope Farms, Woerden, the Netherlands) for 6 weeks. Group sizes were determined statistically before experimentation.

\section{Hematology, Cholesterol Levels, and Autopsy}

Blood was obtained by cardiac puncture and collected into EDTA-containing tubes. Hematological parameters were determined using a ScilVetabc plus (Scil Animal Care Company B.V., Viernheim, Germany). Cholesterol and triglyceride levels in the plasma were determined according to the manufacturer's protocols (CHOD-PAP; Roche Diagnostics, Mannheim, Germany). Autopsy was performed on $n=10$ animals per group, and $>20$ organs were analyzed macroscopically and microscopically on paraffinembedded, hematoxylin and eosin-stained sections $(4 \mu \mathrm{m})$.

\section{Flow Cytometry}

Spleen and lymph nodes were harvested in ice-cold phosphatebuffered saline. Single cell suspensions were prepared and filtered through a $70-\mu \mathrm{m}$ mesh. Spleens were digested using 1 $\mathrm{mg} / \mathrm{mL}$ collagenase D and $20 \mu \mathrm{g} / \mathrm{mL}$ DNaseI (both Roche Diagnostics). Blood and spleens were treated with red blood cell 
A

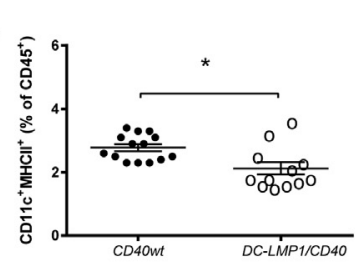

C

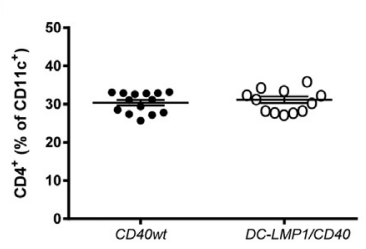

E

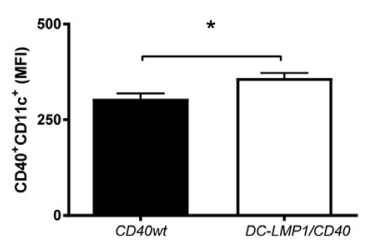

G

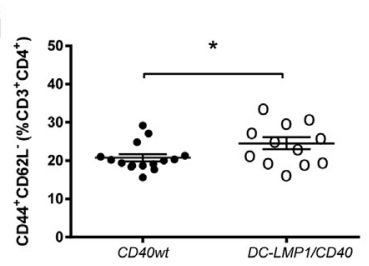

I

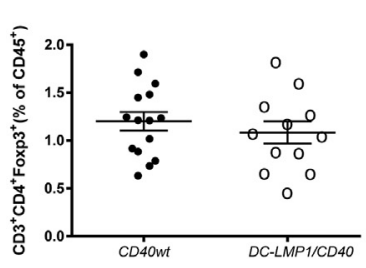

Figure $2 D C-L M P 1 / C D 40 / L_{d l r^{-/-}}$chimeras display DC and T-cell activation. Flow cytometric analysis of $D C S(\mathbf{A}-\mathbf{F})$ and $T$ cells $(\mathbf{G}-\mathbf{I})$ from spleens of CD40wt/Ldlr $r^{-1-}$ and DC-LMP1/CD40/Ldlr $r^{-/}$chimeras. A-D: Fraction of conventional DCs $\left(\mathrm{CD} 11 \mathrm{c}^{+} \mathrm{MHCII}^{+}\right)$of all $\mathrm{CD} 45^{+}$cells $(\mathbf{A})$, fraction of $\mathrm{CD} 8 \alpha^{+} \mathrm{DCs}$ within the $\mathrm{CD} 11 \mathrm{c}^{+}$population (B), fraction of $\mathrm{CD} 4^{+}$ $\mathrm{DCs}$ within the $\mathrm{CD} 11 \mathrm{c}^{+}$population $(\mathbf{C})$, and fraction of $\mathrm{CD} 8 \alpha^{-} \mathrm{CD} 4^{-} \mathrm{DCs}$ within the $\mathrm{CD}_{11 \mathrm{C}^{+}}$population (D). E and F: MFI of CD40 (E) and CD80 (F) on the $\mathrm{CD}_{11 \mathrm{C}^{+}}$population. $\mathbf{G}$ and $\mathbf{H}$ : Fraction of $\mathrm{CD}^{+} \mathrm{CD}_{4} 4^{+}(\mathbf{G})$ and $\mathrm{CD}^{+} \mathrm{CD}_{4} 4^{+}(\mathbf{H})$ effector $\mathrm{T}$ cells in spleen. I: Fraction of $\mathrm{CD}^{+} \mathrm{CD}^{+}{ }^{+} \mathrm{Foxp}^{+}$ cells from leukocytes. Data are expressed as means \pm SEM. $n=14$ to 15 CD40wt/Ldlr ${ }^{-/-}(\mathbf{A}-\mathbf{I}) ; n=12$ DC-LMP1/CD40/Ldlr ${ }^{-/-}(\mathbf{A}-\mathbf{I}) .{ }^{*} P<0.05$. MFI, mean fluorescence intensity.

lysis buffer. Staining against CD45, CD3, CD4, CD8, CD44, CD62L, FoxP3, CD25, CD11c, MHCII, and CD80 used fluorochrome-labeled antibodies (BD Bioscience, Franklin Lakes, NJ, and BioLegend, San Diego, CA). Nonspecific binding was prevented using the $\mathrm{Fc}$ receptor blocking antibody CD16/32 (BD Bioscience). Intracellular staining was performed using the Fixation/Permeabilization Solution kit (BD Bioscience). Flow cytometry was performed using a FACSCanto II (BD Bioscience).

\section{Atherosclerosis}

Upon sacrifice, aortic roots and aortic arches containing their main branch points were dissected, fixed in $1 \%$ paraformaldehyde, processed, and then sectioned. Twenty consecutive sections were selected for histological analysis and stained for hematoxylin and eosin, Picrosirius Red for collagen, Mac3 for macrophages, Ly-6G for neutrophils (BD Bioscience), arginase 1 for M2 macrophages (Thermo Fisher Scientific, Waltham, MA), or inducible nitric oxide synthase (iNOS) for M1 macrophages (Abcam, Cambridge, UK). Morphometric analyses were performed (P.K. and E.L.) using the LAS software version 4.1 (Leica, Wetzlar, Germany), as described before, while blinded for experimental group distribution. ${ }^{19}$ iNOS and arginase 1 stainings were scored on a 1 to 5 scale by two independent investigators (P.K. and E.L.), on the basis of the intensity of the staining pattern.

\section{Statistics}

Unless stated otherwise, the values in all analyses are expressed as means \pm SEM. Differences between groups were analyzed by unpaired $t$-test or $U$-test in the absence of Gaussian distribution, with a significance level of $P<0.05$. Correlations between lipid levels and plaque area were tested using Pearson's correlation coefficient.

\section{Results}

\section{Constitutive Active CD40 Signaling in DCs Causes Neutrophilia}

$D C-L M P 1 / C D 40 / L d l r^{-/}$chimeras had normal blood leukocyte counts (Figure 1A). However, flow cytometric analysis revealed that $D C-L M P 1 / C D 40 / L d l r^{-1-}$ chimeras had pronounced neutrophilia, accompanied by an increase in the percentage of Ly6 $\mathrm{C}^{\text {high }}$ monocytes (Figure 1, B and C).

\section{Constitutive Active CD40 Signaling in DCs Results in DC} and T-Cell Activation

Spleens and lymph nodes of DC-LMP1/CD4O/Ldlr ${ }^{-/}$chimeras showed a slight, but significant, reduction in the fraction of conventional CD11 $\mathrm{c}^{+} \mathrm{MHCII}^{+}$DCs (Figure 2A). These conventional DCs were polarized less toward CD8 $\mathrm{a}^{+}$DCs and more toward $\mathrm{CD}^{-} \mathrm{CD}^{-} \mathrm{DCs}$, whereas the fraction of $\mathrm{CD} 4^{+}$ DCs did not change (Figure 2, B-D). DCs and T cells from spleens and lymph nodes of the DC-LMP1/CD40/Ldlr ${ }^{-1}$ chimeras displayed elevated activation status with increased CD40 and CD80 expression on DCs (Figure 2, E and F). Concomitantly, there was increased abundance of splenic CD44 ${ }^{+} \mathrm{CD} 2 \mathrm{~L}^{-}$effector memory CD4 ${ }^{+} \mathrm{T}$ cells (Figure $2 \mathrm{G}$ ) and a trend toward increased CD44 ${ }^{+} \mathrm{CD}_{2} \mathrm{~L}^{-}$effector memory $\mathrm{CD}^{+}$T cells $(P=0.05)$ (Figure $\left.2 \mathrm{H}\right)$ demonstrating enhanced immune activation in DC-LMP1/CD40/Ldlr ${ }^{-/}$chimeras. Finally, regulatory T-cell numbers in spleen were found to be 


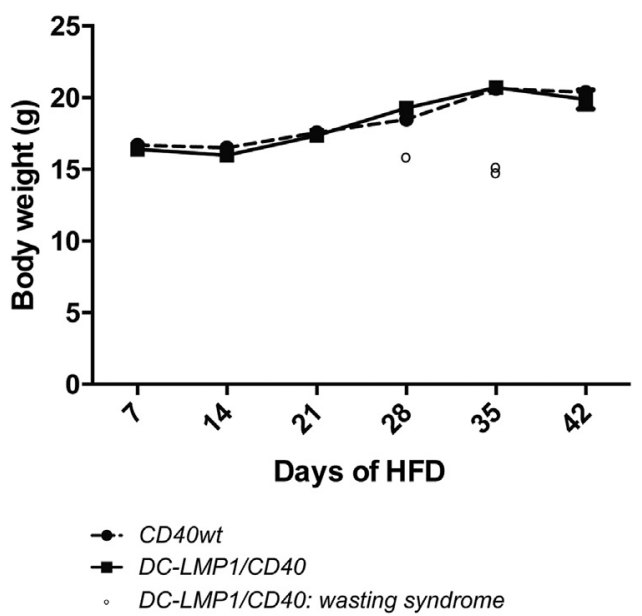

Figure 3 Some $D C-L M P 1 / C D 40 / \mathrm{Ldlr}^{-/-}$chimeras develop wasting syndrome. Body weight of CD40wt/Ldlr ${ }^{-/}$and DC-LMP1/CD40/Ldlr ${ }^{-1-}$ chimeras during the experiment. Open circles represent the mice suffering from wasting syndrome that had to be sacrificed before the end of the experiment. $n=3$ mice with wasting syndrome; $n=15 \mathrm{CD} 40 \mathrm{wt} / \mathrm{Ldll}^{-/-}$ and $D C-L M P 1 / C D 40 / L_{d l r^{-}}-$chimeras. HFD, high-fat diet.

similar between groups (Figure 2I). Similar results were obtained from lymph nodes of $D C-L M P 1 / C D 40 / \mathrm{Ldlr}^{-1-}$ chimeric mice $\left(\mathrm{CD} 4{ }^{+} \mathrm{CD} 44^{+} \mathrm{CD}_{2} \mathrm{~L}^{-}\right.$: CD40wt $14.6 \pm 0.9 \%$ versus $D C-L M P 1 / C D 40 \quad 16.7 \quad \pm 1.0 \%, \quad P=0.08$ $\mathrm{CD}^{+} \mathrm{CD}^{+} 4^{+} \mathrm{CD}_{22} \mathrm{~L}^{-}:$CD40wt $3.8 \pm 0.1 \%$ versus $D C$-LMPI/ $C D 405.1 \pm 0.3 \%, P<0.05)$.

\section{Constitutive Active CD40 Signaling in DCs Reduces Atherosclerosis}

During the 6-week, high-cholesterol diet feeding period, body weights did not differ between the 2 groups. Nonetheless, during the fourth or fifth week of diet feeding, in total 3 of $15 D C-L M P 1 / C D 40 / L d l r^{-/}$chimeras had to be sacrificed because of wasting syndrome and excessive weight loss ( $>8 \%$ body weight in 1 week) (Figure 3). None of the CD40wt/Ldlr ${ }^{-/-}$chimeras died.

Remarkably, constitutively active CD40 signaling in DCs caused a strong reduction in plasma cholesterol and triglyceride levels (Figure 4). DC-LMP1/CD40/Ldlr ${ }^{-1}$ chimeras displayed a strong decrease in atherosclerotic plaque area in the aortic root (Figure 4). When plotting plaque area against cholesterol and triglyceride levels of all individual mice included in the study, a significant negative correlation between plaque area and plasma cholesterol levels (Figure 4) and plaque area and triglyceride levels $(r=0.46$, $P=0.03$ ) was found, suggesting that the decrease in plaque area in $D C-L M P 1 / C D 40 / \mathrm{Ldlr}^{--}$chimeras is caused by the decrease in lipid levels.

Moreover, plaques of $D C-L M P 1 / C D 40 / \mathrm{Ldlr}^{-/-}$chimeras only displayed early atherosclerosis, characterized by the presence of intimal xanthomas and intimal thickenings (Figure 5A), whereas $\mathrm{CD} 40 \mathrm{wt} / \mathrm{Ldlr}^{-1-}$ chimeras had developed advanced atherosclerosis with fibrous cap atheromata (Figure 5B). No changes in plaque collagen content (Figure 5C) or neutrophil infiltration (Figure 5D) were observed, but due to the absence of necrotic cores, plaques of $\mathrm{CD} 40 \mathrm{ca} / \mathrm{Ldlr}^{-1-}$ chimeras displayed a relative increase in macrophage content (Figure 5, E and F). Further analysis of plaque phenotype revealed no differences in macrophage skewing toward iNOS-positive macrophages (M1 phenotype) or arginase-1-positive macrophages (M2 phenotype) between groups (Figure 5, G-J).

In the aortic arch, a similar phenomenon was observed. Although the aortic arch contained small intimal xanthomas at this stage, DC-LMP1/CD40/Ldlr ${ }^{-1-}$ chimeras displayed an $88.6 \%$ decrease in atherosclerosis $\left(C D 40 w t / \mathrm{Ldlr}^{-1-}\right.$ $22,076 \pm 3763 \mu^{2}$ versus DC-LMP1/CD40/Ldlr ${ }^{-1}$ $2511 \pm 1256 \mu \mathrm{m}^{2}$ ).

\section{Constitutive Activation of CD40 Signaling in DCs Causes Colitis}

High-cholesterol diet-fed DC-LMP1/CD40/Ldlr ${ }^{-/-}$chimeras developed severe inflammation of the gastrointestinal tract. Histopathological examination of gastrointestinal specimens revealed massive infiltration of leukocytes in the tunica muscularis, the submucosa, and mucosa resulting in dysplasia of the villous epithelium in DC-LMP1/CD40/ $\mathrm{Ldll}^{-/-}$chimeras (Figure 6, A-G), mimicking inflammatory
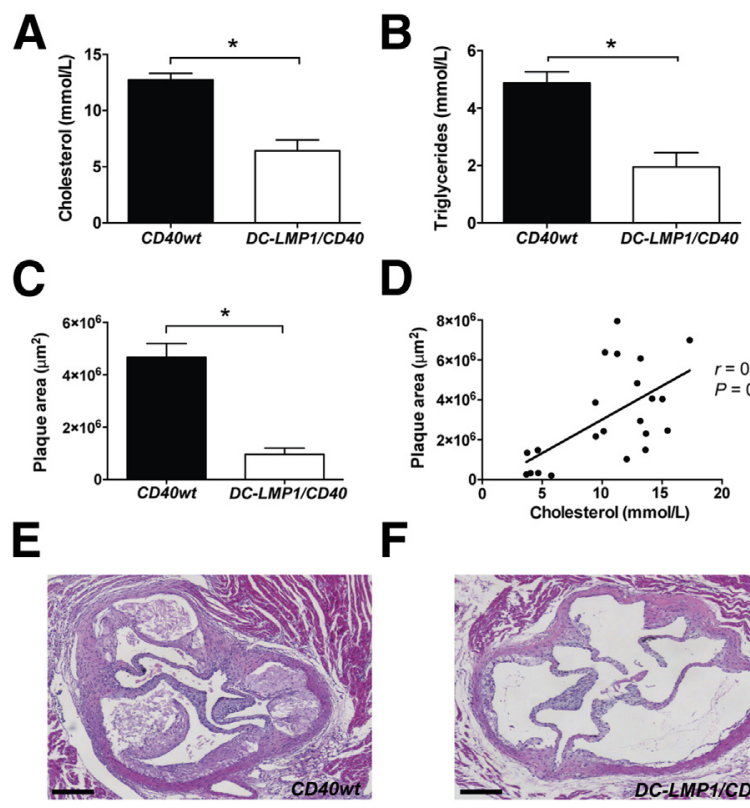

D

Figure 4 Constitutive overexpression of CD40 signaling in DCs reduces plasma cholesterol and atherosclerosis. A: Plasma cholesterol levels of CD40wt/Ldlr ${ }^{-/-}$and DC-LMP1/CD40/Ldlr ${ }^{-/-}$chimeras. B: Plasma triglyceride levels of CD4Owt/Ldlr ${ }^{-1-}$ and DC-LMP1/CD40/Ldlr ${ }^{-/}$chimeras. C: Plaque area of the aortic root of CD40wt/Ldlr ${ }^{-}$and DC-LMP1/CD40/Ldlr ${ }^{-}$ chimeras. D: Correlation of plasma cholesterol levels and plaque area of all mice studied (both genotypes). E and F: Representative photographs of hematoxylin and eosin-stained aortic root sections of CD40wt/Ldlr ${ }^{-1-}(\mathbf{E})$ and $D C-L M P 1 / C D 40 /$ Ldlr $^{-/}$(F) chimeras. Data are expressed as means \pm SEM. $n=12$ DC-LMP1/CD40/Ldlr ${ }^{-/}$chimera (A-D); $n=15$ CD40wt/Ldlr ${ }^{-1-}$ chimera (A-D). ${ }^{*} P<0.05$. Scale bars: $100 \mu \mathrm{m}$ (E and $\left.\mathbf{F}\right)$. 
A

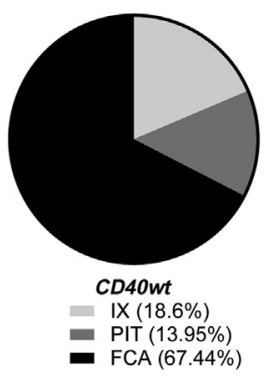

D

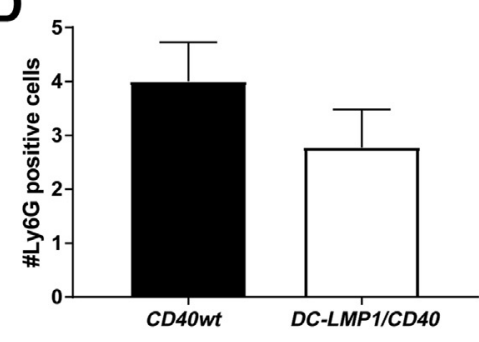

G

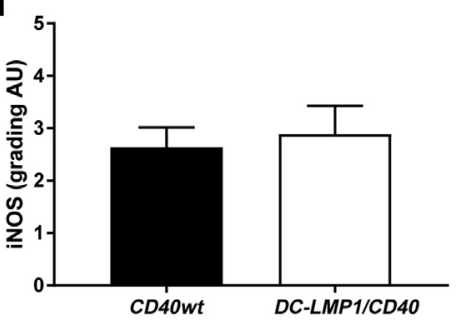

B

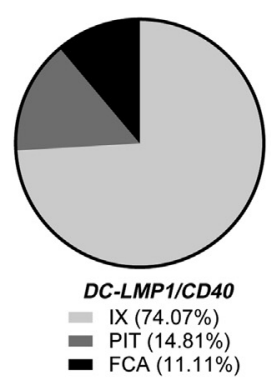

E

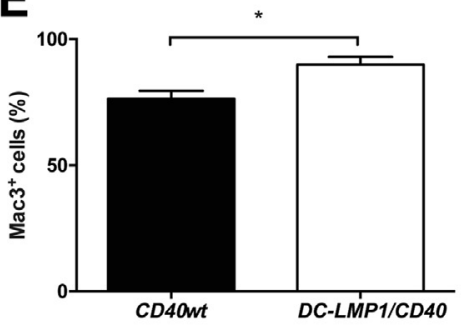

$\mathrm{H}$

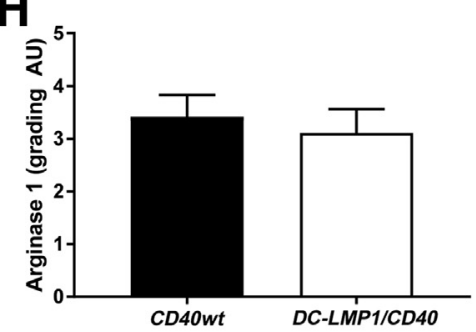

C

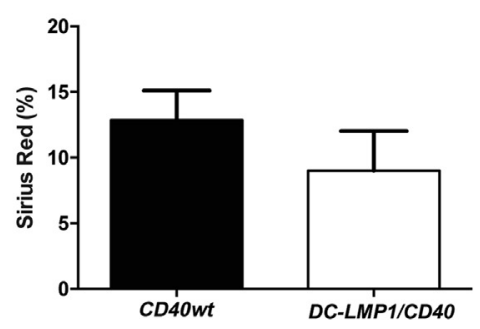

$\mathbf{F}$

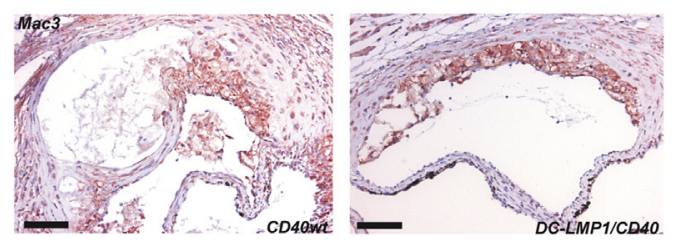

Figure 5 Plaque phenotypic characterization. A and B: Classification of atherosclerotic plaques of CD40wt/ $\mathrm{Ldlr}^{-/-}$(A) and DC-LMP1/CD40/Ldlr ${ }^{-1-}$ (B) chimeras. C: Plaque Sirius Red content, reflecting collagen content. D: Plaque Ly6G-positive neutrophil counts. E: Plaque macrophage content. F: Representative photographs of Mac3 staining of CD40wt/Ldlr ${ }^{-/}$and DC-LMP1/CD40/ $\mathrm{Ldlr}^{-/-}$chimeras. G and H: Plaque scoring for iNOS (G) and Arginase 1 (H). I and J: Representative photographs of iNOS and Arg1 stainings of CD4Owt/Ldlr ${ }^{-/}$(I) and DC-LMP1/CD40/Ldlr ${ }^{-/}$(J) chimeras. Data are expressed as means \pm SEM. $n=8$ to 12 DC-LMP1/CD40/Ldlr chimera (A-E, G, H); $n=11$ to 15 CD40wt/Ldlr/chimera $(\mathbf{A}-\mathbf{E}, \mathbf{G}, \mathbf{H}) .{ }^{*} P<0.05$. Scale bars $=70 \mu \mathrm{m}(\mathbf{F}, \mathbf{I}$, and J). FCA, fibrous cap atheroma; IX, intimal xanthoma; PIT, pathological intimal thickening.

bowel disease (IBD). This phenomenon was most prominent in the caecum but could also be found in the small and large intestines. Interestingly, both plasma cholesterol (Figure $6 \mathrm{H}$ ) and triglyceride levels $(\mathrm{r}=-0.66, P=0.03)$ were inversely correlated with disease severity in DC-LMP1/CD40/Ldlr ${ }^{-/-}$ chimeras, suggesting that IBD causes lipid malabsorption. No abnormalities or enhanced leukocyte infiltration was observed in any of the other organs examined.

\section{Discussion}

The costimulatory molecule CD40 has a profound role in DC maturation, antigen presentation, and DC-dependent
T-cell expansion. ${ }^{2,24}$ In our previous studies using $C D 40^{-1-}$ mice or $C D 40^{-1-}$ bone marrow chimeras, we showed an altered immune status in mice lacking CD40. ${ }^{14,15,17,19}$ These changes were accompanied by a reduction in effector $\mathrm{T}$ cells and $\mathrm{CD}^{-} \mathrm{CD}^{-} \mathrm{DCs}$, and an antiinflammatory monocyte/macrophage profile as the most prominent features, which resulted in a profound reduction of atherosclerosis. ${ }^{19}$

Here, the $D C-L M P 1 / C D 40$ model with $C^{2} 11 c^{+}$DCspecific expression of the latent membrane protein LMP-1 fused to CD40 intracellular domain conferring constitutive activation of CD40 signaling ${ }^{20}$ was used to study the impact of DC CD40 signaling on atherosclerosis. We confirm that 

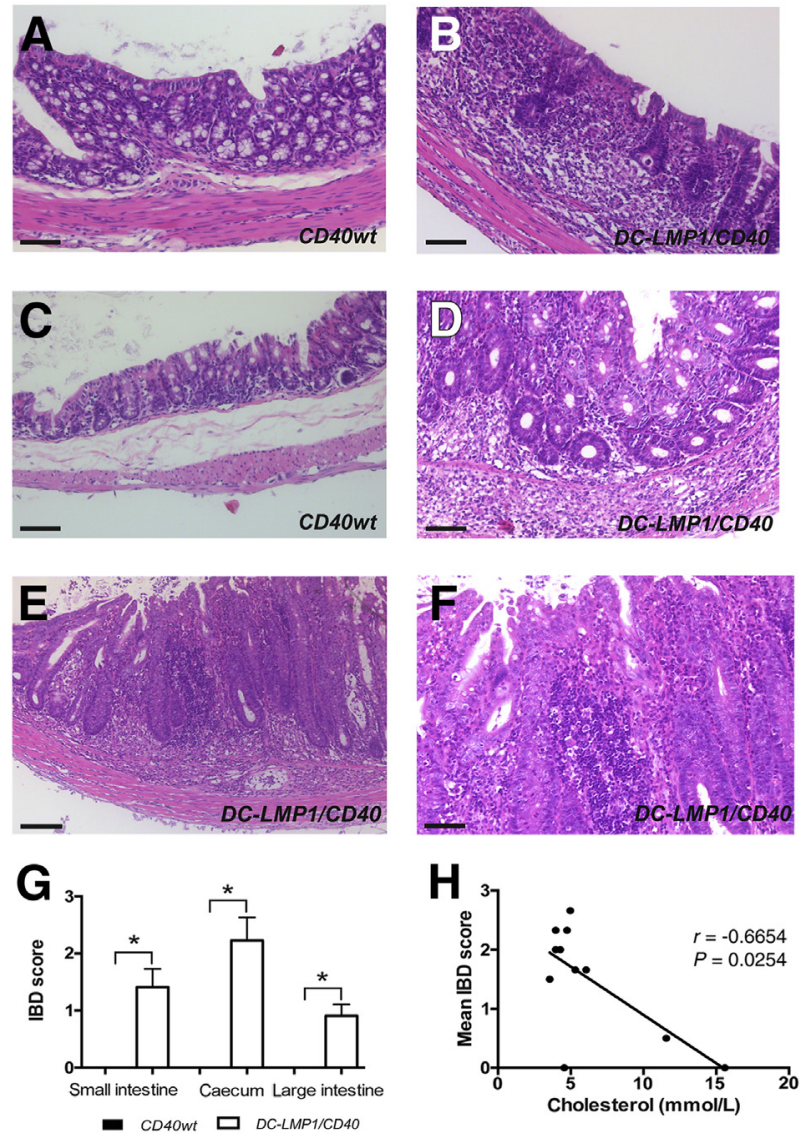

Figure 6 Constitutive overexpression of CD40 signaling in DCs results in IBD. A: Hematoxylin- and eosin (H\&E)-stained section of the colon of a CD40wt/Ldlr ${ }^{-1}$ chimera showing a normal, intact colon. B: H\&E-stained section of the colon of a $D C-L M P 1 / C D 40 / L_{d l r^{-}}{ }^{-}$chimera showing severe inflammation in mucosa and submucosa, and villous degradation. C: H\&Estained section of the cecum of a CD4OWt/Ldlr ${ }^{-/-}$chimera showing a normal, intact cecum. D: H\&E-stained section of the cecum of a DC-LMP1/ $\mathrm{CD} 40 / \mathrm{Ldlr}^{-/}$chimera with massive IBD: leukocyte infiltrate penetrating the mucosa and submucosa, showing destruction of crypts. $\mathbf{E}$ and $\mathbf{F}$ : Overview (E) and close-up (F) of the cecum of a DC-LMP1/CD40/Ldlr ${ }^{-/}$ chimera and displaying influx of leukocytes and lymphocytes in the mucosa, submucosa and villous epithelium. G: IBD scores, reflecting disease severity in CD4Owt/ $\mathrm{Ldlr}^{-/-}$chimeras (no disease, black bars), and DC-LMP1/ CD40/ $\mathrm{Ldlr}^{-1-}$ chimeras (white bars). H: Correlation between plasma cholesterol levels and IBD severity in DC-LMP1/CD $40 / \mathrm{Ldll}^{-/-}$chimeras. Data are expressed as means \pm SEM (G). $n=10 D C-L M P 1 / C D 40 / L d l r$ chimera (G and $\mathbf{H}) .{ }^{*} P<0.05$. Scale bars: $50 \mu \mathrm{m}(\mathbf{A}-\mathbf{D}$, and $\mathbf{F}) ; 100 \mu \mathrm{m}(\mathbf{E})$.

constitutive activation of CD40 on DCs results in expansion and increased frequency of $\mathrm{CD} 4^{+}$and $\mathrm{CD} 8^{+}$effector T cells in blood and lymphoid organs, although $\mathrm{CD} 4^{+} \mathrm{Foxp}^{+}$ regulatory $\mathrm{T}$ cell numbers in the spleen did not differ significantly between groups. Moreover, DCs from $D C$ LMP1/CD40/Ldlr ${ }^{-1}$ chimeras had augmented costimulatory capacity with increased MHCII and CD80 expression.

Surprisingly, $D C-L M P 1 / C D 40 / L d l r^{-/-}$chimeras also featured increased neutrophil counts. In search for the cause of this neutrophilia, autopsy revealed inflammation of the gastrointestinal tract, with signs of IBD, especially in the cecum, which were only present in $D C-L M P 1 / C D 40 /$ $\mathrm{Ldlr}^{-\prime-}$ chimeras.

Given this strong proinflammatory phenotype, we were surprised to observe reduced atherosclerosis, as well as reduced plasma cholesterol and triglyceride levels, in the DC-LMP1/CD $40 / \mathrm{Ldlr}^{-/-}$chimeras. However, as previously reported for patients suffering from IBD, colitis can induce a decrease in total and LDL-associated cholesterol levels due to reduced intestinal lipid uptake. ${ }^{25}$

The involvement of CD40 signaling in IBD was reported before. ${ }^{26}$ Scid mice reconstituted with propathogenic $\mathrm{CD} 4 \mathrm{RB}^{\text {high }} \mathrm{CD}^{+} \mathrm{T}$ cells develop colitis with high expression of CD40 and CD40L in the inflamed intestinal mucosa. ${ }^{27}$ Upon treatment with CD40L-blocking antibody, colitis in these mice was prevented or cured. ${ }^{27}$ By contrast, treatment of immune-deficient Rag-deficient mice with an agonistic CD40 antibody caused colitis and wasting syndrome ${ }^{28}$ highlighting the importance of CD40-CD40L interactions in the pathogenesis of colitis. Similarly, mucosal biopsies of IBD patients contained increased numbers of $\mathrm{CD}_{40} 0^{+}$and $\mathrm{CD} 40 \mathrm{~L}^{+}$cells, while these patients also displayed elevated plasma levels of soluble CD40L. ${ }^{29,30}$ Furthermore, both CD40 expression in ulcerative colitis and plasma soluble CD40L levels were found to be proportional to disease activity. ${ }^{31}$ Moreover, direct antagonistic treatment of CD40 decreased disease activity in a clinical setting. ${ }^{32,33}$ In a recent separate study using the $D C$-LMP1/CD40 mouse, Barthels et $\mathrm{al}^{21}$ showed that colitis in this model occurs due to a reduction of $\mathrm{CD} 103^{+}$DCs and $\mathrm{ROR} \gamma \mathrm{t}^{+} \mathrm{Helios}^{-}$induced regulatory T-cells. This triggers a Th1/Th17-driven autoimmune reaction against the microbiome leading to (fatal) colitis.

In addition to the profound T-cell and DC activation, a reduction in the amount of conventional CD11 ${ }^{+} \mathrm{MHCII}^{+}$ DCs in lymphoid organs of DC-LMP1/CD40/Ldlr ${ }^{-1-}$ chimeras was observed. Interestingly, DC numbers and their (immune) activity are implicated in the regulation of plasma cholesterol concentrations. Increased DC lifespan, as seen upon BclII overexpression, not only led to increased DC numbers and activation status, but also was accompanied by decreased cholesterol levels. ${ }^{34}$ Accordingly, transient depletion of DCs by diphtheria toxin treatment in $c d 11 c$ DTR/Apoe ${ }^{-/-}$mice produced a transient increase in plasma cholesterol levels. ${ }^{34}$ Dendritic cells are able to accumulate lipids during hypercholesterolemic conditions, ${ }^{35}$ and defective cholesterol efflux can lead to inflammasome activation and proinflammatory signaling in DCs. ${ }^{36}$ Currently, it remains unclear how cholesterol levels are controlled by conventional DC; however, DCs might promote lipoprotein uptake and clearance from the circulation. ${ }^{34}$

In the present study however, we could not confirm these results because our mice had fewer DCs and lower cholesterol levels, suggesting that the observed colitis and subsequent systemic inflammation, rather than factors such as DC quantity, affect cholesterol metabolism in our model. 
The original aim of our study was to investigate the role of CD40 on DCs in atherosclerosis. By examining DCspecific CD40 overexpression instead of deletion, we aimed to further elucidate the specific role of CD40 in the contribution of DCs to atherosclerosis, as well as learn more about the systemic effects CD40 activation has via DCs on other cell types in hypercholesterolemic conditions. Although constitutive expression of CD40 on DCs in $\mathrm{Ldlr}^{-/-}$mice resulted in the expected inflammatory phenotype of $\mathrm{T}$ cells and DCs, DC-LMP1/CD40/Ldlr ${ }^{-/-}$chimeras demonstrated an unexpected reduced atherosclerotic burden. DC-LMPI/ $\mathrm{CD} 40 / \mathrm{Ldlr}^{-/-}$chimeras developed IBD-like pathology accompanied by a reduction in lipid levels outweighing the overall proinflammatory milieu. Although the large difference in lesion area makes it difficult to draw strong conclusions on the basis of plaque phenotyping data, there did not appear to be strong effects of DC CD40 signaling with regard to the skewing of lesion macrophages, the infiltration of neutrophils, or the generation of regulatory $\mathrm{T}$ cells. Thus, the most likely pathophysiological mechanism by which continuous CD40 activation on DCs reduces lesion size remains via the induction of IBD.

As such, this renders this model less suitable to study the role of DC-specific CD40 in atherosclerosis.

\section{Acknowledgments}

P.K., T.S., C.B., B.L., and H.W. conceived and performed experiments; P.K., T.S., B.L., and M.G. conceived experiments and analyzed data; C.B., R.B., L.B., and D.A. performed experiments; E.B., T.B., C.W., N.G., and E.L. supervised experiments; E.L. designed the project and wrote most of the paper; all authors were involved in writing the paper and had final approval of the submitted version.

\section{References}

1. Libby P, Lichtman AH, Hansson GK: Immune effector mechanisms implicated in atherosclerosis: from mice to humans. Immunity 2013, 38:1092-1104

2. Legein B, Temmerman L, Biessen EAL, Lutgens E: Inflammation and immune system interactions in atherosclerosis. Cell Mol Life Sci 2013, 70:3847-3869

3. Ketelhuth DFJ, Hansson GK: Adaptive response of T and B cells in atherosclerosis. Circ Res 2016, 118:668-678

4. Tabas I, Bornfeldt KE: Macrophage phenotype and function in different stages of atherosclerosis. Circ Res 2016, 118:653-667

5. Cybulsky MI, Cheong C, Robbins CS: Macrophages and dendritic cells: partners in atherogenesis. Circ Res 2016, 118:637-652

6. Millonig G, Niederegger H, Rabl W, Hochleitner BW, Hoefer D, Romani N, Wick G: Network of vascular-associated dendritic cells in intima of healthy young individuals. Arterioscler Thromb Vasc Biol 2001, 21:503-508

7. Paulson KE, Zhu S-N, Chen M, Nurmohamed S, Jongstra-Bilen J, Cybulsky MI: Resident intimal dendritic cells accumulate lipid and contribute to the initiation of atherosclerosis. Circ Res 2010, 106:383-390

8. Subramanian M, Tabas I: Dendritic cells in atherosclerosis. Semin Immunopathol 2014, 36:93-102
9. Ait-Oufella H, Sage AP, Mallat Z, Tedgui A: Adaptive (T and B cells) immunity and control by dendritic cells in atherosclerosis. Circ Res 2014, 114:1640-1660

10. Mildner A, Jung S: Development and function of dendritic cell subsets. Immunity 2014, 40:642-656

11. Hedrick CC: Lymphocytes in atherosclerosis. Arterioscler Thromb Vasc Biol 2015, 35:253-257

12. Chen L, Flies DB: Molecular mechanisms of T cell co-stimulation and co-inhibition. Nat Rev Immunol 2013, 13:227-242

13. Croft M: The role of TNF superfamily members in T-cell function and diseases. Nat Rev Immunol 2009, 9:271-285

14. Engel D, Seijkens T, Poggi M, Sanati M, Thevissen L, Beckers L, Wijnands E, Lievens D, Lutgens E: The immunobiology of CD154-CD40TRAF interactions in atherosclerosis. Semin Immunol 2009, 21:308-312

15. Lutgens E, Gorelik L, Daemen MJ, de Muinck ED, Grewal IS, Koteliansky VE, Flavell RA: Requirement for CD154 in the progression of atherosclerosis. Nat Med 1999, 5:1313-1316

16. Mach F, Schönbeck U, Sukhova GK, Atkinson E, Libby P: Reduction of atherosclerosis in mice by inhibition of CD40 signalling. Nature 1998, 394:200-203

17. Lutgens E, Cleutjens KB, Heeneman S, Koteliansky VE, Burkly LC, Daemen MJ: Both early and delayed anti-CD40L antibody treatment induces a stable plaque phenotype. Proc Natl Acad Sci U S A 2000, 97 : 7464-7469

18. Schönbeck U, Sukhova GK, Shimizu K, Mach F, Libby P: Inhibition of CD40 signaling limits evolution of established atherosclerosis in mice. Proc Natl Acad Sci U S A 2000, 97:7458-7463

19. Lutgens E, Lievens D, Beckers L, Wijnands E, Soehnlein O, Zernecke A, Seijkens T, Engel D, Cleutjens J, Keller AM, Naik SH, Boon L, Oufella HA, Mallat Z, Ahonen CL, Noelle RJ, de Winther MP, Daemen MJ, Biessen EA, Weber C: Deficient CD40-TRAF6 signaling in leukocytes prevents atherosclerosis by skewing the immune response toward an antiinflammatory profile. J Exp Med 2010, 207:391-404

20. Hömig-Hölzel C, Hojer C, Rastelli J, Casola S, Strobl LJ, Müller W, Quintanilla-Martinez L, Gewies A, Ruland J, Rajewsky K, ZimberStrobl U: Constitutive CD40 signaling in B cells selectively activates the noncanonical NF-kappaB pathway and promotes lymphomagenesis. J Exp Med 2008, 205:1317-1329

21. Barthels C, Ogrinc A, Steyer V, Meier S, Simon F, Wimmer M, Blutke A, Straub T, Zimber-Strobl U, Lutgens E, Marconi P, Ohnmacht C, Garzetti D, Stecher B, Brocker T: CD40-signalling abrogates induction of ROR $\gamma \mathrm{t}+$ Treg cells by intestinal CD103+ DCs and causes fatal colitis. Nat Commun 2017, 8:14715

22. Caton ML, Smith-Raska MR, Reizis B: Notch-RBP-J signaling controls the homeostasis of CD8- dendritic cells in the spleen. J Exp Med 2007, 204:1653-1664

23. Rastelli J, Hömig-Hölzel C, Seagal J, Müller W, Hermann AC, Rajewsky K, Zimber-Strobl U: LMP1 signaling can replace CD40 signaling in $\mathrm{B}$ cells in vivo and has unique features of inducing classswitch recombination to IgG1. Blood 2008, 111:1448-1455

24. Bäck M, Weber C, Lutgens E: Regulation of atherosclerotic plaque inflammation. J Intern Med 2015, 278:462-482

25. Agouridis AP, Elisaf M, Milionis HJ: An overview of lipid abnormalities in patients with inflammatory bowel disease. Ann Gastroenterol 2011, 24:181-187

26. Senhaji N, Kojok K, Darif Y, Fadainia C, Zaid Y: The contribution of CD40/CD40L axis in inflammatory bowel disease: an update. Front Immunol 2015, 6:529

27. Liu Z, Geboes K, Colpaert S, Overbergh L, Mathieu C, Heremans H, de Boer M, Boon L, D’Haens G, Rutgeerts P, Ceuppens JL: Prevention of experimental colitis in SCID mice reconstituted with CD45RBhigh CD4+ T cells by blocking the CD40-CD154 interactions. J Immunol 2000, 164:6005-6014

28. Annacker O, Coombes JL, Malmstrom V, Uhlig HH, Bourne T, Johansson-Lindbom B, Agace WW, Parker CM, Powrie F: Essential role for CD103 in the T cell-mediated regulation of experimental colitis. J Exp Med 2005, 202:1051-1061 
29. Liu Z, Colpaert S, D'Haens GR, Kasran A, de Boer M, Rutgeerts P, Geboes K, Ceuppens JL: Hyperexpression of CD40 ligand (CD154) in inflammatory bowel disease and its contribution to pathogenic cytokine production. J Immunol 1999, 163:4049-4057

30. Battaglia E, Biancone L, Resegotti A, Emanuelli G, Fronda GR, Camussi G: Expression of CD40 and its ligand, CD40L, in intestinal lesions of Crohn's disease. Am J Gastroenterol 1999, 94:3279-3284

31. Polese L, Angriman I, Cecchetto A, Norberto L, Scarpa M, Ruffolo C, Barollo M, Sommariva A, D'Amico DF: The role of CD40 in ulcerative colitis: histochemical analysis and clinical correlation. Eur J Gastroenterol Hepatol 2002, 14:237-241

32. Danese S, Sans M, Scaldaferri F, Sgambato A, Rutella S, Cittadini A, Piqué JM, Panes J, Katz JA, Gasbarrini A, Fiocchi C: TNF-alpha blockade down-regulates the CD40/CD40L pathway in the mucosal microcirculation: a novel anti-inflammatory mechanism of infliximab in Crohn's disease. J Immunol 2006, 176:2617-2624

33. Kasran A, Boon L, Wortel CH, Hogezand RA, Schreiber S, Goldin E, Boer M, Geboes K, Rutgeerts P, Ceuppens JL: Safety and tolerability of antagonist anti-human CD40 Mab ch5D12 in patients with moderate to severe Crohn's disease. Aliment Pharmacol Ther 2005, 22: $111-122$

34. Gautier EL, Huby T, Saint-Charles F, Ouzilleau B, Pirault J, Deswaerte V, Ginhoux F, Miller ER, Witztum JL, Chapman MJ, Lesnik P: Conventional dendritic cells at the crossroads between immunity and cholesterol homeostasis in atherosclerosis. Circulation 2009, 119:2367-2375

35. Packard RRS, Maganto-Garcia E, Gotsman I, Tabas I, Libby P, Lichtman $\mathrm{AH}: \mathrm{CD} 11 \mathrm{c}(+)$ dendritic cells maintain antigen processing, presentation capabilities, and CD4(+) T-cell priming efficacy under hypercholesterolemic conditions associated with atherosclerosis. Circ Res 2008, 103:965-973

36. Westerterp M, Gautier EL, Ganda A, Molusky MM, Wang W, Fotakis P, Wang N, Randolph GJ, D'Agati VD, Yvan-Charvet L, Tall AR: Cholesterol accumulation in dendritic cells links the inflammasome to acquired immunity. Cell Metab 2017, 25: 1294-1304.e6 